\title{
Activación Sónica Versus Ultrasónica de EDTA al $10 \%$ para Remoción de Barrillo Dentinario en el Tercio Apical del Canal Radicular
}

\author{
Sonic Versus Ultrasonic Activation of $10 \%$ EDTA for \\ Smear Layer Removal in the Root Canal Apical Third
}

Valeria Isabel Pérez De Arce Carrasco*; Pamela Arlenne Rodríguez Olivares* \& Diego Echeverri Caballero*

PEREZ DE ARCE, C. V. I.; RODRÍGUEZ, O. P. A. \& ECHEVERRI, C. D. Activación sónica versus ultrasónica de EDTA al 10\% para remoción de barrillo dentinario en el tercio apical del canal radicular. Int. J. Odontostomat., 8(1):153-159, 2014.

RESUMEN: El objetivo de este estudio fue evaluar la efectividad de la activación sónica y ultrasónica del ácido etilendiaminotetraacético (EDTA) al 10\% para remover barrillo dentinario en el tercio apical y el grado de erosión producido sobre las paredes del canal radicular. Se realizó un estudio cuantitativo experimental in vitro. Se instrumentaron 40 dientes manualmente con la técnica step-back hasta lima maestra número 55 y fueron asignados a 4 grupos según protocolo de irrigación final: I: agua destilada estéril sin activación, II: EDTA 10\% sin activación por 30 segundos, III: EDTA 10\% activado sónicamente por 30 segundos y IV: EDTA 10\% activado ultrasónicamente por 30 segundos. Las muestras fueron observadas en MEB a una magnificación de 5000X. Se evaluó la presencia de barrillo dentinario remanente y grado de erosión según criterios de Torabinejad et al. Para el análisis estadístico se utilizó la prueba de Kruskal-Wallis y Post Hoc de Tukey con un nivel de significancia 5\% ( $p=0,05)$. El grupo I (agua sin activación) presentó mayor cantidad de barrillo que el resto de los grupos $(p<0,01)$. En cuanto a la presencia de barrillo, entre los grupos II (EDTA sin activación), III (EDTA con sonido) y IV (EDTA con ultrasonido) no hubo diferencias estadísticamente significativas. En cuanto a la erosión, el grupo II presentó una mayor erosión que el resto siendo significativa con los grupos I y III $(p=0,006)$. Entre los grupos I, III y IV no hubo diferencias estadísticamente significativas. La activación sónica y ultrasónica del EDTA $10 \%$ no produjo una remoción de barrillo significativamente superior al compararlo con la irrigación convencional, pero sí hubo diferencias en cuanto a erosión, siendo menor con la activación sónica.

PALABRAS CLAVE: EDTA, activación de irrigantes, irrigación ultrasónica pasiva, irrigación sónica pasiva, erosión dentinaria, remoción de barrillo.

\section{INTRODUCCIÓN}

Durante la instrumentación de canales radiculares es inevitable la formación de barrillo dentinario (Violich \& Chandler, 2010), el cual está constituido por una capa amorfa de material orgánico e inorgánico, remanentes dentinarios, tejido pulpar, procesos odontoblásticos y en dientes infectados, bacterias y sus productos (Lui et al., 2007). Su remoción es necesaria durante el tratamiento endodóntico (Gu et al., 2009) ya que puede albergar bacterias, interferir con los materiales de obturación, limitar la efectividad de irrigación y medicación al interior de los túbulos dentinarios (Violich \& Chandler) y resultar en una inflamación perirradicular persistente (Gu et al.).

La irrigación es parte esencial del debridamiento ya que permite la limpieza de los canales más allá de lo que se logra con la instrumentación (Gu et al.). Los irrigantes deben disolver el contenido orgánico e inorgánico, siendo el método más efectivo el uso alternado de EDTA con $\mathrm{NaOCl}$ (Dadresanfar et al., 2011), que ha reportado una completa remoción en los tercios coronal y medio (Torabinejad et al., 2003). No obstante, la remoción en el tercio apical sigue siendo impredecible (Kuah et al., 2009). Los métodos actuales para la remoción del barrillo dentinario incluyen sustancias químicas, y agitación sónica y ultrasónica, ninguna totalmente eficaz a lo largo de todo el canal radicular (Violich \& Chandler). La activación ultrasónica de los irrigantes se ha asociado directamente a un aumento en la efectividad de la limpieza de los canales radiculares, pero presenta limitaciones como la inhibición del efecto de cavitación cuando el instrumento

*Escuela de Odontología, Universidad Austral de Chile, Valdivia, Chile. 
contacta las paredes del canal siendo menos efectiva en el tercio apical. La activación sónica ofrece ventajas como evitar el riesgo de transporte, perforación y desgaste excesivo en las paredes del canal aunque la velocidad de vibración es inferior. En consecuencia los estudios en que se comparan los resultados obtenidos con el uso de estas dos técnicas de activación muestran resultados contrastantes (Kanter et al., 2011).

Existe la necesidad de estandarizar los protocolos de activación de irrigantes para realizar comparaciones y determinar el ideal. El objetivo del estudio fue evaluar la efectividad en la remoción de barrillo dentinario y erosión producida en el tercio apical del canal radicular al activar la irrigación final con EDTA al $10 \%$ con sonido y ultrasonido y compararlo con la irrigación convencional.

\section{MATERIAL Y MÉTODO}

Estudio experimental in vitro donde se recolectaron 40 dientes permanentes extraídos en las clínicas Odontológicas de la Universidad Austral de Chile (UACh), sin restos periodontales. Se almacenaron en alcohol metílico $75 \%$ refrigerados a $4^{\circ} \mathrm{C}$. El muestreo fue por conveniencia debido a la accesibilidad y las características que tenían que cumplir los dientes. Se incluyeron dientes unirradiculares, con raíz recta, formación apical completa y que al examen radiográfico se observó un solo canal. Se excluyeron dientes con dislaceración, más de un canal, reabsorción radicular, canales previamente tratados o con fractura radicular. Se tomaron 2 radiografías por diente, en sentido mesio-distal y vestíbulo-palatino/lingual con películas velocidad D, tamaño 2 (Kodak Ultra Speed, Eastman Kodak Company, Nueva York, EE.UU.) y equipo de rayos $X$ intraoral (Planmeca Intra, Planmeca Oy, Helsinki, Finlandia) a 64 kV, 8 mA y tiempo de exposición de 0,320 s. Para el revelado de las películas se utilizó una máquina reveladora de rayos $X$ automática (Dürr Periomat Plus, Dürr Dental, Bietigheim-Bissingen, Alemania). Una vez terminada la selección se tallaron surcos guías con fresa transmetálica en sentido longitudinal de la raíz y en sentido transversal, sin perforar el canal radicular. Se cortaron todos los dientes a nivel coronal dejando 20 $\mathrm{mm}$ de longitud dentaria para estandarizar la longitud de trabajo en $19,5 \mathrm{~mm}$, manteniéndose a 0,5 $\mathrm{mm}$ del foramen apical, medidos con una lima $20 \mathrm{~K}$ (Dentsply Maillefer, Ballaigues, Suiza) y regla de endodoncia milimetrada.
Los dientes seleccionados fueron divididos de manera aleatoria simple en 4 grupos de 10 dientes cada uno según protocolo de irrigación. El grupo control negativo fue irrigado con agua destilada estéril sin activación y el grupo control positivo fue irrigado con $\mathrm{NaOCl}$ al $5,25 \%$ más EDTA al $10 \%$ sin activación. Los grupos experimentales fueron irrigados con $\mathrm{NaOCl}$ al $5,25 \%$ más EDTA al 10\% con activación sónica o ultrasónica, según corresponda. Los grupos fueron los siguientes:

Grupo I: Irrigación con $9 \mathrm{ml}$ de agua destilada estéril durante la instrumentación.

Grupo II: Irrigación con $9 \mathrm{ml}$ de $\mathrm{NaOCl}$ al 5,25\% durante la instrumentación, más irrigación final con $1 \mathrm{ml}$ de EDTA al $10 \%$ por 30 segundos.

Grupo III: Irrigación con $9 \mathrm{ml}$ de $\mathrm{NaOCl}$ al 5,25\% durante la instrumentación, más irrigación final con $1 \mathrm{ml}$ de EDTA al $10 \%$ con activación sónica por 30 segundos. Grupo IV: Irrigación con $9 \mathrm{ml}$ de $\mathrm{NaOCl}$ al 5,25\% durante la instrumentación, más irrigación final con $1 \mathrm{ml}$ de EDTA al 10\% con activación ultrasónica por 30 segundos.

Todos los dientes fueron sumergidos hasta el límite cervical en un vaso dappen con alginato (Plastalgin, Septodont, Francia) y se instrumentaron de manera manual con la técnica step-back, utilizando limas K (Dentsply Maillefer, Ballaigues, Suiza), desde la No15 a la N55 de manera secuencial; cada lima se utilizó con movimientos de fuerza balanceada (recíproco), irrigando con $1 \mathrm{ml}$ de $\mathrm{NaOCl} 5,25 \%$ en cada cambio de instrumento hasta conseguir $9 \mathrm{ml}$ de irrigación durante la fase de preparación. La irrigación final de cada grupo fue según protocolo indicado. Posteriormente se irrigó con $10 \mathrm{ml}$ de agua destilada estéril y se secó con conos de papel (Dentsply Maillefer, Ballaigues, Suiza). La irrigación se realizó con jeringa monoject (Kendall, Mansfield MA, USA) introducida de manera pasiva a 2 milímetros sobre la longitud de trabajo durante la instrumentación. La irrigación final con activación sónica se realizó utilizando Endoactivator (Dentsply Maillefer, Ballaigues, Suiza) con inserto azul (30/0.6) a $1 \mathrm{~mm}$ de la longitud de trabajo, vibrando a $10.000 \mathrm{~Hz}$ y la ultrasónica con unidad ultrasónica Varios 350 (NSK Nakanishi, Kanuma-shi, Japón) con lima ultrasónica Ufiles №20 (NSK Nakanishi, Kanuma-shi, Japón) a 1 mm de la longitud de trabajo, vibrando a $30.000 \mathrm{~Hz}$.

Terminada la instrumentación e irrigación, los dientes fueron seccionados con cizalla, a nivel de los surcos guías tallados, de los cuales una muestra del tercio apical de la raíz fue preparada para ser montada y observada en microscopio electrónico de barrido (mo- 
delo Leo 420, Inglaterra). Dos examinadores calibrados $(\mathrm{k}=90 \%)$, cegados al régimen de irrigación, evaluaron la superficie del canal radicular en el tercio apical, tomando una microfotografía panorámica de la muestra a $160 x$ y una de la zona más representativa a una magnificación de 5000x. La calibración de los examinadores se realizó en dos sesiones teóricas dirigidas por el investigador a cargo, más dos sesiones prácticas donde se discutieron microfotografías con distintos grados de erosión y presencia de barrillo dentinario en dientes instrumentados, un mes previo a el primer análisis de las muestras. El acuerdo intra e inter examinador se calculó mediante la prueba estadístico Kappa-Cohen.

Los examinadores evaluaron la presencia de barrillo dentinario remanente $y$ grado de erosión según los criterios de Torabinejad et al.

Cantidad de barrillo dentinario:

1: Ausencia de barrillo dentinario. Ausencia de barrillo dentinario en la superficie del canal radicular. Todos los túbulos estaban limpios y abiertos.

2: Moderado barrillo dentinario. Ausencia de barrillo dentinario en la superficie del canal radicular, pero los túbulos contienen detritus.

3: Abundante barrillo dentinario. Barrillo dentinario cubre la superficie del canal radicular y los túbulos.

Grado de erosión:

1: Sin erosión. Todos los túbulos con apariencia y tamaño normal.

2: Erosión moderada. La dentina peritubular fue erosionada.

3: Erosión severa. La dentina intertubular fue destruida y hay conexión entre los túbulos.

Los datos fueron tabulados y analizados con el programa estadístico SPSS Statistics versión 20.0 (IBM., Chicago, EE.UU). Para el análisis estadístico, se utilizó la prueba de Kruskal- Wallis y el Post Hoc de Tukey con un nivel de significancia $5 \%(p=0,05)$.

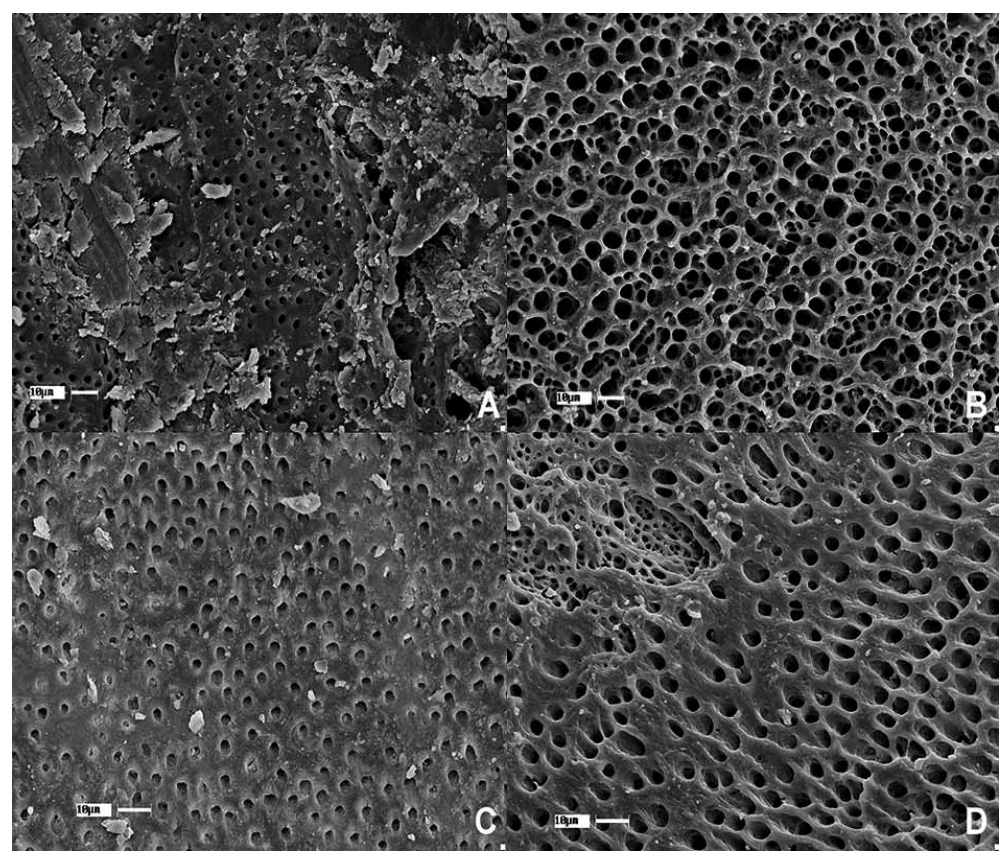

Fig. 1. Microfotografías de cada grupo de estudio a 5000X, donde se obtuvo la menor cantidad de barrillo remanente. Según los criterios de Torabinejad et al. (2003) se clasifican en: A (grupo I) cantidad de barrillo 2 y grado de erosión 1, B (grupo II) cantidad de barrillo 1 y grado de erosión 3, C (grupo III) cantidad de barrillo 1 y grado de erosión 1 y D (grupo IV) cantidad de barrillo 1 y grado de erosión 2.

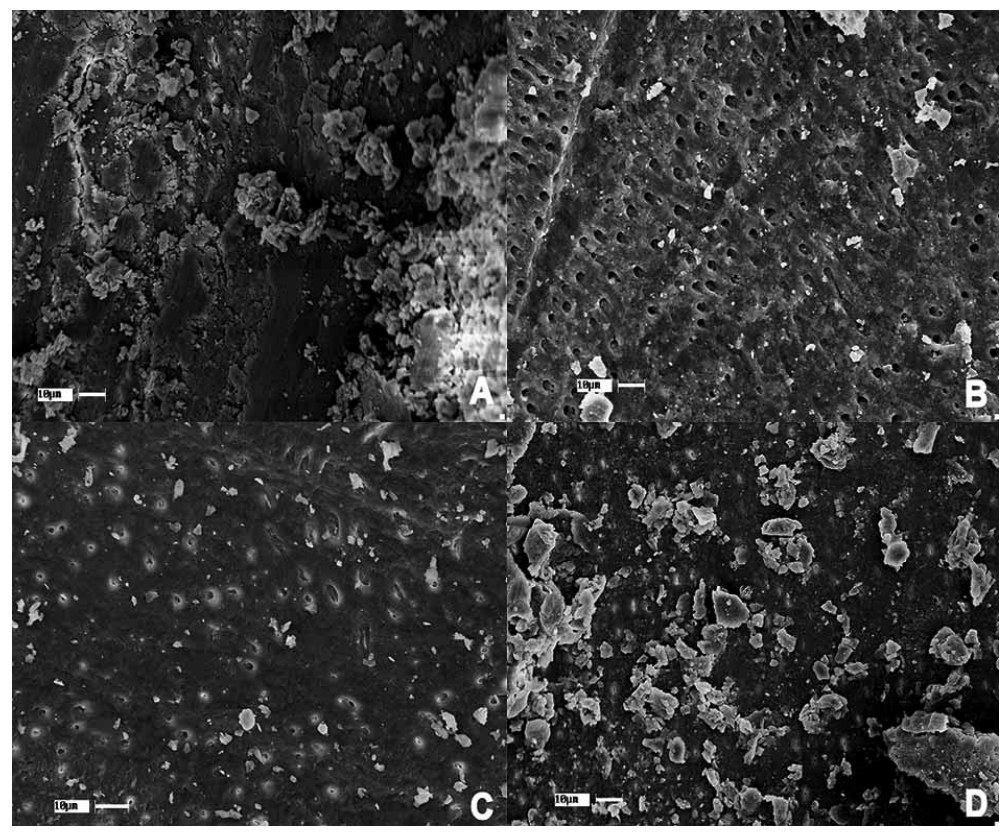

Fig. 2. Microfotografías de cada grupo de estudio a 5000X, donde se obtuvo la mayor cantidad de barrillo remanente. Según los criterios de Torabinejad et al. (2003) se clasifican en: A (grupo I) cantidad de barrillo 3 y grado de erosión 1, B (grupo II) cantidad de barrillo 2 y grado de erosión 1, C (grupo III) cantidad de barrillo 2 y grado de erosión 1 y D (grupo IV) cantidad de barrillo 3 y grado de erosión 1. 


\section{RESULTADOS}

Las Figuras 1 y 2 muestras las imágenes características de la superficie del canal radicular tras ejecutar los diferentes regímenes de irrigación. El análisis estadístico de los datos hecho mediante la prueba de Kruskal Wallis mostró diferencias estadísticamente significativas con un valor de $p<0,01$ entre los grupos para eliminación y un $p=0,09$ para erosión. Complementando el análisis con un test post hoc de Tukey, se evidenció que en cuanto a la presencia de barrillo dentinario remanente en las superficies del canal radicular con los diferentes protocolos de irrigación, el grupo de EDTA sin activar mostró una mayor limpieza de las superficies, pero no hubo una diferencia estadísticamente significativa con los grupos activados sónica y ultrasónicamente $(p=0,970$ y 0,810 , respectivamente).

Entre los grupos en que se utilizó activación de los irrigantes (grupos III y IV) el grupo III mostró una menor cantidad de barrillo remanente (Figs. 1C y 2C), aunque la diferencia no fue estadísticamente significativa $(p=0,970)$. En el grupo control negativo se observó abundante acumulación de barrillo en la superficie del canal y obstruyendo los túbulos dentinarios (Figs. $1 \mathrm{~A}$ y $2 \mathrm{~A}$ ) siendo la diferencia con respecto a los demás grupos estadísticamente significativa $(p=<0,01)$.

Con respecto al grado de erosión de la dentina peri e intertubular en las paredes del canal radicular, el grupo que mostró una mayor cantidad de muestras severamente erosionadas (registro 3) fue el grupo de EDTA sin activar (Fig. 1B), siendo la diferencia respecto al grupo control negativo y al activado sónicamente, estadísticamente significativa $(p<0,01)$ aunque no lo fue con el activado ultrasónicamente $(p=0,166)$.

Al comparar entre los grupos activados, el grupo con activación sónica no exhibió ningún nivel de erosión a diferencia del grupo con activación ultrasónica, donde el $10 \%$ de las muestras presentó erosión (registro 3), pero esta diferencia carece de significancia $(p=0,499)$. Entre los activados y el control negativo, que no presentó ningún grado de erosión, tampoco hubo diferencias significativas $(p=0,499)$. El porcentaje de muestras que exhibieron mayor nivel de limpieza (registro 1) en cada grupo, y el mayor grado de erosión (registro 3) se encuentran ilustrados en la Tabla I.
La comparación entre los grupos en cuanto a la cantidad de barrillo remanente y grado de erosión, con sus correspondientes valores de $\mathrm{P}$, se resumen en las Tablas II y III respectivamente.

Tabla I. Porcentaje de superficies limpias (registro 1) y erosión (registro 3) en los grupos del I al IV.

\begin{tabular}{lcc}
\hline Grupo & Limpieza & Erosión \\
\hline I & $0 \%(\mathrm{a})$ & $0 \%(\mathrm{a})$ \\
II & $70 \%(\mathrm{~b})$ & $50 \%(\mathrm{~b})$ \\
III & $60 \%(\mathrm{~b})$ & $0 \%(\mathrm{a})$ \\
IV & $50 \%(\mathrm{~b})$ & $10 \%(\mathrm{a})(\mathrm{b})$ \\
\hline
\end{tabular}

Letra diferente indica diferencias estadísticamente significativas.

Tabla II. Valor de P para cantidad de barrillo dentinario remanente entre grupos del I al IV.

\begin{tabular}{ccc}
\hline Grupo & Grupo de comparación & Valor de \\
\hline I & II, III y IV & $\mathrm{P}<0,001$ \\
II & III & $\mathrm{P}=0,970$ \\
III & IV & $\mathrm{P}=0,810$ \\
IV & III y IV & $\mathrm{P}=0,970$ \\
& II & $\mathrm{P}=0,810$ \\
III & $\mathrm{P}=0,970$ \\
\hline
\end{tabular}

Tabla III. Valor de P para grado de erosión dentinaria entre grupos del I al IV

\begin{tabular}{lcc}
\hline Grupo & Grupo de comparación & Valor de $\mathbf{P}$ \\
\hline \multirow{4}{*}{ I } & II, & $\mathrm{P}<0,01$ \\
& III & $\mathrm{P}=1,0$ \\
II & IV & $\mathrm{P}=0,499$ \\
& I y III & $\mathrm{P}<0,01$ \\
III & IV & $\mathrm{P}=0,166$ \\
& II & $\mathrm{P}<0,01$ \\
IV & IV & $\mathrm{P}=0,499$ \\
& I y III & $\mathrm{P}=0,499$ \\
& II & $\mathrm{P}=0,166$ \\
\hline
\end{tabular}

\section{DISCUSIÓN}

El propósito de esta investigación fue evaluar el efecto de la activación sónica y ultrasónica del EDTA al $10 \%$ en la remoción del barrillo dentinario y la erosión dentinaria producida en las paredes del canal radicular a nivel del tercio apical.

Los resultados indican que todos los grupos mostraron diferentes niveles de barrillo remanente y erosión dentinaria, como se puede observar al comparar entre las microfotografías de las Figuras 1 y 2 , 
donde se evidencia significativamente menos barrillo en el grupo irrigado con EDTA al 10\% sin activación (Figs. 1B y 2B), seguido por el grupo con EDTA al 10\% con activación sónica (Figs. $1 \mathrm{C}$ y $2 \mathrm{C}$ ), el grupo con EDTA al 10\% con activación ultrasónica (Figs. 1D y 2D) y finalmente el grupo irrigado con agua destilada (Figs. $1 \mathrm{~A}$ y $2 \mathrm{~A}$ ). Al comparar la presencia de barrillo dentinario entre los grupos con irrigación activada (III y IV, sónica y ultrasónica, respectivamente) no se observan diferencias estadísticamente significantes. Esto puede deberse a que la activación agita el irrigante (EDTA) tan vigorosamente que puede provocar la formación de burbujas o ser dispersado fuera del canal. Como consecuencia el irrigante deja de estar en contacto con la superficie dentinaria por cortos periodos de tiempo y no logra el efecto esperado.

La activación sónica (vibraciones de gran amplitud y baja frecuencia) mostró mejores resultados aunque no estadísticamente significativos $(p=0,970)$, en comparación con la ultrasónica (vibraciones de poca amplitud y alta frecuencia) (Blank-Gonçalves et al., 2011). Esto podría explicarse si se considera que el inserto sónico no se inactiva al contactar con las paredes del canal, lo que sí ocurre con la lima ultrasónica, evento que puede reducir su eficiencia al utilizarla en el tercio apical, debido a que la anatomía es más compleja y de menores dimensiones, inhibiéndose con facilidad el efecto de cavitación.

En cuanto al grado de erosión dentinaria, los grupos activados mostraron menor deterioro superficial en comparación con la irrigación convencional. Esto puede ser consecuencia del movimiento constante y burbujeante del irrigante al interior del canal. En la irrigación tradicional, el quelante tiene más tiempo de contacto con el sustrato sobre el que actúa. Es interesante notar que la erosión fue mayor, pero no significativa, en la irrigación ultrasónica en comparación con la sónica. Aun cuando la irrigación ultrasónica es pasiva, la alta frecuencia de vibración y la rigidez del inserto pueden generar el mayor deterioro de las paredes del canal.

Los resultados de esta investigación fueron contrastantes con algunos reportes de la literatura que afirma las ventajas de los métodos de activación ultrasónica (Kuah et al.; Lui et al.) y sónica (Caron et al., 2010).

Dadrensafar et al. encontró un incremento de erosión significativo al activar sónicamente en el tercio coronal y medio, pero no encontró diferencias en el tercio apical. En cuanto a la remoción de barrillo dentinario encontró que la activación del EDTA presentó mejores resultados aunque la diferencia no fue estadísticamente significativa. Blank-Gonçalves et al. encontró que los sistemas de activación eliminan significativamente más barrillo dentinario que la irrigación convencional. Resultados similares se obtuvieron en el estudio de Al-Ali et al. (2012) donde la irrigación ultrasónica pasiva presentó significativamente menos barrillo dentinario remanente que la agitación manual del irrigante (EDTA y $\mathrm{NaOCl}$ ). Ribeiro et al. (2012) encontró que ninguno de los protocolos de agitación de los irrigantes removió completamente la capa de barrillo en canales radiculares aplanados, pero los sistemas de agitación asistidos por máquinas, entre ellos el ultrasonido, removieron más barrillo que las técnicas manuales.

Recíprocamente, Saber \& Hashem (2011) encontró mayor remoción de barrillo al agitar manualmente el EDTA que de forma ultrasónica pasiva y UrozTorres et al. (2010) informó que el Endoactivator no mostró mejoras en la remoción de barrillo al compararlo con la irrigación convencional con $\mathrm{NaOCl}$ y EDTA. Rödig et al. (2010) no encontró diferencias significativas en la remoción de barrillo en el tercio apical de raíces curvas con activación de irrigantes y en el estudio de Mayer et al. (2002) la activación ultrasónica de los irrigantes no redujo la cantidad de barrillo dentinario a pesar del mayor tiempo de activación (1 minuto).

En cuanto a la metodología del presente estudio, el inyectar el irrigante con jeringa permite controlar el volumen y profundidad de penetración de la aguja y el flujo de irrigante en la región apical del sistema de canales (van der Sluis et al., 2007). La utilización de la técnica de instrumentación step-back desde la lima 15 a la 55 facilita la adecuada penetración de irrigantes al tercio apical, ya que según Torabinejad et al. se requiere ampliar el canal por sobre una lima 30 para lograr este objetivo. Esta técnica permite aumentar el diámetro y la conicidad del CANAL radicular lo que influye en la eficacia de la irrigación ultrasónica pasiva para remover el barrillo dentinario según van der Sluis et al.

Los resultados de una investigación in vitro deben extrapolarse a la clínica con precaución. Se enfrentan varias dificultades al desarrollar un estudio de este tipo. Por una parte, son muchas las variables a considerar dentro del diseño metodológico que pueden afectar de manera significativa los resultados observados (tiempo de activación, concentración de irrigantes, variaciones anatómicas de los dientes se- 
leccionados, etc.). Se debe considerar además que al estudiar una superficie con gran magnificación (MEB) es posible obtener diferentes observaciones sobre el mismo campo de observación lo que puede llevar a diferentes interpretaciones de los resultados.

No hay consenso respecto al volumen óptimo, tiempo de aplicación o método de activación del irrigante (Blank-Gonçalves et al.), por lo que se insta a seguir investigando para determinar los ideales y estandarizar los protocolos de irrigación.

De acuerdo a lo observado en esta investigación, la activación ultrasónica y sónica del EDTA al $10 \%$ no produce una mejora significativa en la remoción de barrillo dentinario al compararla con la utilización de EDTA en forma convencional, pero sí consigue menor erosión de la dentina de las paredes del canal radicular.

\section{AGRADECIMIENTOS}

A todos quienes han hecho posible esta investigación, en especial al Dr. Ricardo Silva Riveros, Ingeniero eléctrico, Académico del Instituto de Anatomía, Histología y Patología de la Universidad Austral de Chile, a cargo del microscopio electrónico de barrido (MEB), por su ayuda, disposición y cooperación en la obtención de las microfotografías, y al Dr. Diego Halabí Robles, Cirujano Dentista, por guiarnos en el ámbito de la estadística.

Este artículo está basado en una tesis presentada en cumplimento parcial de los requisitos para la obtención del grado de Licenciado en Cirugía Dental en la Escuela de Odontología de la Universidad Austral de Chile, Valdivia, Chile. (en noviembre de 2013.)

PEREZ DE ARCE, C. V. I.; RODRÍGUEZ, O. P. A. \& ECHEVERRI, C. D. Sonic versus ultrasonic activation of 10\% EDTA for smear layer removal in the root canal apical third. Int. J. Odontostomat., 8(1):153-159, 2014.

ABSTRACT: The purpose of this study was to observe the effect of passive sonic and ultrasonic irrigation with $10 \%$ Ethilenediaminotetracethic acid (EDTA) on smear layer of the apical third of instrumented root canals as well as the level of erosion produced on the canal walls. For this quantitative experimental in vitro study 40 teeth were hand instrumented with step back technique until master file num 55 and divided in four groups according to the final irrigation protocol: I: distilled water without activation, II: 10\% EDTA for 30 seconds without activation, III: 10\% EDTA sonic activated for 30 seconds, IV: $10 \%$ EDTA ultrasonic activated for 30 seconds. Samples were prepared for MEB observation at 5000X. The presence of smear layer and erosion on dentine walls was quantified according to Torabinejad et al. criteria. Data were submitted at Kruskal-Wallis test and Tukey Post Hoc with a significance level of $5 \%(P=0.05)$. When evaluating the smear layer presence, there were no statistically significant differences between the groups II, III and IV. Group I had a highest amount of smear layer $(p<0.01)$. As for erosion, group II showed higher erosion than the others, being significant with groups I and III $(p=0.006)$. Among groups I, III and IV there were not statistically significant differences. Sonic and ultrasonic activation of EDTA 10\% did not produce a significantly superior removal of smear layer when compared with conventional irrigation. Erosion of root canal walls was significantly lower after sonic activation.

KEY WORDS: EDTA, irrigant activation, passive ultrasonic irrigation, passive sonic irrigation, dentin erosion, smear layer removal.

\section{REFERENCIAS BIBLIOGRÁFICAS}

Al-Ali, M.; Sathorn, C. \& Parashos, P. Root canal debridement efficacy of different final irrigation protocols. Int. Endod. J., 45(10):898-906, 2012.

Blank-Gonçalves, L. M.; Nabeshima, C. K.; Martins, G. H. \& Machado, M. E. Qualitative analysis of the removal of the smear layer in the apical third of curved roots: conventional irrigation versus activation systems. J. Endod., 37(9):1268-71, 2011.
Caron, G.; Nham, K.; Bronnec, F. \& Machtou, P. Effectiveness of different final irrigant activation protocols on smear layer removal in curved canals. J. Endod., 36(8):1361-6, 2010.

Dadresanfar, B.; Khalilak, Z.; Delvarani, A.; Mehrvarzfar, P.; Vatanpour, M. \& Pourassadollah, M. Effect of ultrasonication with EDTA or MTAD on smear layer, debris and erosion scores. J. Oral Sci., 53(1):31-6, 2011. 
Gu, L. S.; Kim, J. R.; Ling, J.; Choi, K. K.; Pashley, D. H. \& Tay, F. R. Review of contemporary irrigant agitation techniques and devices. J. Endod., 35(6):791-804, 2009.

Kanter, V.; Weldon, E.; Nair, U.; Varella, C.; Kanter, K.; Anusavice, K. \& Pileggi, R. A quantitative and qualitative analysis of ultrasonic versus sonic endodontic systems on canal cleanliness and obturation. Oral Surg. Oral Med. Oral Pathol. Oral Radiol. Endod., 112(6):809-13, 2011.

Kuah, H. G.; Lui, J. N.; Tseng, P. S. \& Chen, N. N. The effect of EDTA with and without ultrasonics on removal of the smear layer. J. Endod., 35(3):393-6, 2009.

Lui, J. N.; Kuah, H. G. \& Chen, N. N. Effect of EDTA with and without surfactants or ultrasonics on removal of smear layer. J. Endod., 33(4):472-5, 2007.

Mayer, B. E.; Peters, O. A. \& Barbakow, F. Effects of rotary instruments and ultrasonic irrigation on debris and smear layer scores: a scanning electron microscopic study. Int. Endod. J., 35(7):582-9, 2002.

Ribeiro, E. M.; Silva-Sousa, Y. T.; Souza-Gabriel, A.; SousaNeto, M. D.; Lorencetti, K. T. \& Silva, S. R. Debris and smear removal in flattened root canals after use of different irrigant agitation protocols. Microsc. Res. Tech., 75(6):781-90, 2012.

Rödig, T.; Döllmann, S.; Konietschke, F.; Drebenstedt, S. \& Hülsmann, M. Effectiveness of different irrigant agitation techniques on debris and smear layer removal in curved root canals: a scanning electron microscopy study. J. Endod., 36(12):1983-7, 2010.

Saber, Sel-D. \& Hashem, A. Efficacy of different final irrigation activation techniques on smear layer removal. J. Endod., 37(9):1272-5, 2011.

Torabinejad, M.; Khademi, A. A.; Babagoli, J.; Cho, Y.; Johnson, W. B.; Bozhilov, K.; Kim, J. \& Shabahang, S. A new solution for the removal of the smear layer. J. Endod., 29(3):170-5, 2003.

Uroz-Torres, D.; González-Rodríguez, M. P. \& Ferrer-Luque, C. M. Effectiveness of the EndoActivator System in removing the smear layer after root canal instrumentation. J. Endod., 36(2):308-11, 2010.

van der Sluis, L.; Versluis, M.; Wu, M. \& Wesselink, P. Passive ultrasonic irrigation of the root canal: a review of the literature. Int. Endod. J., 40(6):415-26, 2007.

Violich, D. \& Chandler, N. The smear layer in endodontics a review. Int. Endod. J., 43(1):2-15, 2010.

\author{
Dirección para Correspondencia: \\ Valeria Pérez De Arce \\ Instituto de Odontoestomatología \\ Universidad Austral de Chile \\ Valdivia \\ CHILE \\ Email: valeria181@gmail.com
}

Recibido : 13-11-2013

Aceptado: 03-02-2014 